\title{
A novel hemoclip applied on patients with duodenal papillary neoplasms during endoscopic papillectomy to prevent post-procedure bleeding
}

\author{
Zheng Ruhua, Wang Lei, Yao Yuling, Li Yunhong, Cao Jun and Zou Xiaoping* \\ Department of Gastroenterology, tThe Affiliated Drum Tower Hospital of Nanjing University Medical School, Nanjing 210008, China
}

\begin{abstract}
Background and aims: Endoscopic papillectomy is becoming first-line treatment of duodenal papillary neoplasms, yet with certain adverse events including postprocedure bleeding. Currently little is known about the effective approach to prevent late on-set hemorrhage. The aim of this study was to evaluate whether hemoclips are helpful in preventing post-procedure bleeding.

Methods: A retrospective study was conducted from August 2007 to October 2016. A total of 58 patients with duodenal papillary neoplasm underwent endoscopic papillectomy were reviewed. Post-procedure complications were recorded, the prevalence of post-operation bleeding was compared between patients used with clips and without, as well as patients used with two different kinds of hemoclips.

Results: The mean age of 58 patients was $58.4 \pm 9.8$ years, and technical success rate was $100 \%$. The pathological results of resected specimens revealed 53 adenomas and 5 adenocarcinomas. One of the 5 adenocarcinomas had positive vertical margin, so that the clinical success rate was $98.3 \%(57 / 58)$. The post-procedure complications are comprised of cholangitis (5/32), pancreatitis (6/32) and bleeding (9/32). No operation related perforation occurred. The incidence of bleeding in patients applied with hemoclips and without was $19.2 \%(5 / 26)$ versus $12.5 \%(4 / 32)$, respectively ( $\mathrm{p}=0.717$, Fisher's exact test). Two different types of hemoclips were used in the 32 patients. The former one was applied from 2007 to 2014, and since 2015, we have been using hemoclips manufactured by Micro-Tech Inc. Nanjing, China. The postprocedure bleeding rate of the two groups of patients was 15.0\% (3/20, the former group) and 8.3\% (1/12, the Micro-Tech group), respectively((p>0.99, Continuity correction of chi-squared test).
\end{abstract}

Conclusion: Endoscopic papillectomy is feasible in patients with papillary adenoma and noninvasive adenocarcinoma. Hemoclips seem to be useful for preventing post-procedure bleeding. The patients used with Micro-Tech hemoclips had lower bleeding rate than with other one, yet without statistical significance. Further investigation with larger study population is needed to evaluate the effectiveness of hemoclips.

\section{Introduction}

Endoscopic resection of duodenal papillary neoplasms-mostly papillary adenoma-is considered as first-line approach other than radical surgery in recent years. Endoscopic therapy enables patients to suffer less pain and recover faster, yet along with occurrence of certain complications during or after the procedure, including bleeding, pancreatitis, perforation, cholangitis, etc. Among the complications mentioned above, the prevalence of post-procedure bleeding is reported to be $10.0 \% \sim 17.3 \%$. Patients with bleeding usually need transfusion and/or endoscopic hemostasis in severe cases. According to literatures, hemostasis measures include argon plasma coagulation(APC), hemoclips and adrenaline injection. However, prophylactic hemostasis modalities are rarely mentioned in previous studies. In our center since 2007, we have been trying to apply metal clips right after resecting the tumor, in order to avoid post-operation bleeding. From 2015, we began to use a novel metal clip, which could be manipulated much more smoothly and freely through working channel of the duodenoscope compared with the former one used before. This study is aimed to evaluate whether the use of hemoclips immediately after resection could help reduce the incidence of late on-set bleeding.

\section{Patients and methods}

A total of 58 patients with duodenal papillary neoplasm underwent endoscopic papillectomy from August 2007 to October 2016 were retrospectively reviewed. All lesions were assessed by biopsy and EUS to assure that they conformed to indication of endoscopic resection. Papillary neoplasms suitable for endoscopic treatment should be confirmed as adenoma by pre-operation biopsy, or adenocarcinoma limited to mucosa and submucosa layer of duodenal wall evaluated by EUS. Tumors growing into common bile duct and/or main pancreatic duct were excluded, which were assessed by EUS.

The operation procedure is as follows: Mixed solution with diluted adrenaline (1:10000), saline and methylene blue is injected into

Correspondence to: Zou Xiaoping, Department of Gastroenterology, The Affiliated Drum Tower Hospital of Nanjing University Medical School, Nanjing 210008, China, Email: 13770771661@163.com

Key words: Endoscopic papillectomy, Duodenal papillary neoplasms, Hemoclip, Post-procedure bleeding

Received: January 03, 2018; Accepted: January 26, 2018; Published: January 30, 2018 
submucosa surround the lesion to lift the lesion. A snare is placed with its tip cephalad to the papilla, then around the whole lesion. The tumor was resected by tightened snare with electricity. If there is wound errhysis after resection, argon plasma coagulation(APC) would be used. Plastic stent in pancreatic duct and/or common bile duct is placed in some patients to reduce risk of post-operation pancreatitis and cholangitis. Hemoclips are set on the anal direction of the wounds to prevent late on-set bleeding in part of the patients (Figure 1). The hemoclips used after 2015 were manufactured by Micro-Tech Inc., Nanjing China. The endoscope system is Olympus Evis Lucera(Olympus Inc., Tokyo, Japan), and the type of duodenoscope is TJF-260V(Olympus Inc., Tokyo, Japan). Written informed consent was obtained from all patients before operation.

Clinical outcomes were reviewed. Technical success rate was defined as tumor removed by either en-bloc or piecemeal resection. Clinical success rate are was defined as no residual tumor tissue found during follow up endoscopy and biopsy. We emphatically recorded the postprocedure bleeding rates of all patients, and made comparison between patients applied with and without hemoclips, as well as patients used the former and Micro-tech hemoclips. The number of clips used was also recorded.

Statistical analysis was conducted with SPSS Statistics version 19.0(SPSS Inc., USA). Category variables were compared using Chisquared test, or Fisher's exact test if the results were not suitable for Chisquared test. Quantitative variables were compared using independent students' $t$ test if they were normally distributed, and Wilcoxon rank sum test was used if the samples were not normally distributed. A p value less than $5 \%$ was considered as statistically significant.

\section{Results}

A total of 58 patients underwent endoscopic resection of duodenal papillary tumors in our center from August 2007 to October 2016, consisting of 37 males and 21 females, with a mean age of $58.4 \pm 9.8$ years old. Technical success was achieved in all patients (58/58). Post-operation pathological results included 53 adenomas and 5 adenocarcinomas, and positive vertical margin appeared in one of the 5 adenocarcinoma specimens, leading to a clinical success rate of $98.3 \%$ (57/58).

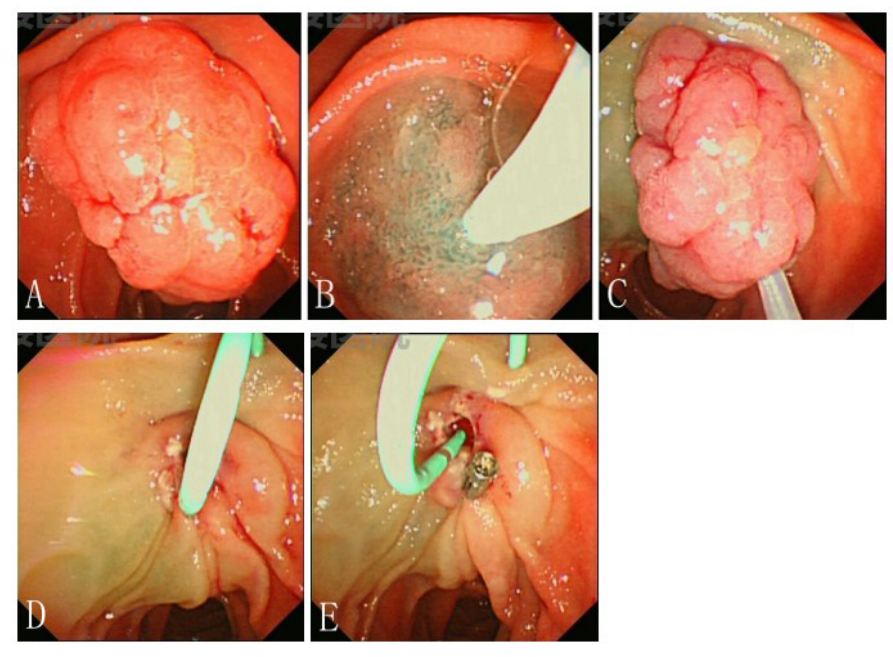

Figure 1. A: Papillary adenoma observed under duodenoscopy; B: Mixed solution was injected into submucosa to lift the lesion; C: A snare was applied to cut the adenoma; D: A plastic stent was placed into main pancreatic duct; E: A metal hemoclip was released on the wound.
Post-procedure complications occurred in 16 patients, with 20 events comprising 5 cholangitis (8.6\%), 6 pancreatitis (10.3\%) and 9 late on-set bleeding (15.5\%). Hemoclips were applied in 32 patients, 4 of which encountered bleeding. The bleeding took place at 12 33 hours after operation (mean time 20hrs); Three out of the 4 bleeding patients received endoscopic hemostasis by electrocoagulation and/or hemoclips for one time, and the other patient had conservative therapy; The other 26 patients had no clips used during operation, 5 of who confronted post-operative bleeding. The bleeding time were $4 \sim 28$ hours after operation (mean time 12.8hrs). Among the 5 bleeding patients, 1 underwent endoscopic hemostasis for one time, and 2 for two times to stop bleeding, another bleeding patient complicated with perforation received surgical therapy, and the last patient had conservative treatment. Between the two groups, the rate of postoperative bleeding exhibited no statistical significant difference (clip group: $12.5 \%$ vs. none clip group: $19.2 \%, \mathrm{p}=0.717$, Fisher's exact test).

Among 32 patients employed hemoclips, the former clips were used in 20 patients and the micro-tech clips were applied in the other 12 patients. $2.35 \pm 1.23$ clips were applied in former clip group, while $1.83 \pm 0.71$ Clips were used in Micro-Tech group. ( $\mathrm{p}=0.256$, Wilcoxon rank sum test). Post-procedure bleeding rate was $15.0 \%(3 / 20)$ and $8.3 \%(1 / 12)$ in former and micro-tech clip groups, respectively $(\mathrm{p}>0.99$, Continuity correction of chi-squared test). All three bleeding patients of the former clip group underwent endoscopic hemostasis operation after bleeding, while the bleeding patient of micro-tech clip group received conservative therapy only.

\section{Discussion}

Duodenal papillary adenoma is rare. According to the previous studies, it occurred in less than $5 \%$ patients with digestive tract neoplasms [1-3]. The clinical manifestations are unspecific unless the growing tumor blocks the orifice of bile and/or pancreatic duct causing obstructive jaundice and/or recurrent pancreatitis. The detection rate is rising during the past decades due to routine EGD examination and ERCP procedure. Papillary adenoma represents similar biological characteristics to colon adenoma which has a malignant potential through adenoma-adenocarcinoma sequence as reported [4]. Earlier studies identified that the transformation risk from adenoma to carcinoma are $25 \% \sim 85 \%$ in sporadic patients and $4 \%$ in familial adenomatous polyposis(FAP) patients [5].

Pancreaticoduodenectomy(PD) is used to be the standard treatment of papillary adenoma; however, the morbidity is high in patients underwent PD [6,7]. Endoscopic papillectomy(EP) is much less invasive alternative procedure compared with $\mathrm{PD}$, which is associated with lower mortality, mortality and decreased cost [8-10]. To cure papillary adenoma endoscopically requires non-residual resection, and its early adverse events and recurrence issues is arousing operators' concern. The most common acute adverse events after EP include bleeding (10\% 17.3\%), pancreatitis $(13.8 \% \sim 20 \%)$ and perforation (1.6\% 7.7\%) [11-13]. In our study, the incidences of pancreatitis and bleeding were similar with the previous studies, while there was no perforation occurred.

To date few literatures have explored prophylactic hemostasis method aimed at treating post-procedure bleeding. We have been applying hemoclips to place on the wound after endoscopic resection, in order to decrease the occurrence rate of post-procedure bleeding. Compared with the group without placing clips, the post-procedure bleeding rate was lower in the group using clips, while without statistical significance. From 2007 to 2014, we employed the former hemoclip in 
20 patients, and 3 patients suffered bleeding after operation $(3 / 20)$. Since 2015 we have been using a novel hemoclip manufactured by Micro-tech Corporation with the same aim. A total of 12 patients were employed with the new clip, and 1 patients encountered post-procedure bleeding (1/12), lower than the former clip group, although with no statistical significance as well.

Comparing with the other hemoclips, the Micro-Tech hemoclip is rotatable and can be repeatedly opening and closing, thus facilitating the exact positioning of the clip. The tail of the clip is shorter, which makes releasing the clip much easier, especially when there is limited space between the tip of endoscope and the lesion. In this study, only 1 out of 12 patient had post-procedure bleeding, suggesting that the Micro-Tech hemoclip is, to some extent, useful for preventing bleeding after endoscopic papillectomy. Our study had limitations due to its small study population and retrospective method. Therefore, a large, blind, multicenter, prospective study is needed for further investigation.

\section{References}

1. Ryan DP, Schapiro RH, Warshaw AL (1986) Villous tumors of the duodenum. Ann Surg 203: 301-306. [Crossref]

2. Gray G, Browder W (1989) Villous tumors of the ampulla of Vater: local resection versus pancreatoduodenectomy. South Med J 82: 917-920. [Crossref]

3. Scarpa A, Capelli P, Zamboni G, Oda T, Mukai K, et al. (1993) Neoplasia of the ampulla of Vater. Ki-ras and p53 mutations. Am J Pathol 142: 1163-1172. [Crossref]
4. Hirota WK1, Zuckerman MJ, Adler DG, Davila RE, Egan J, et al. (2006) ASGE guideline: the role of endoscopy in the surveillance of premalignant conditions of the upper GI tract. Gastrointest Endosc 63: 570-580. [Crossref]

5. El Hajj II, Coté GA (2013) Endoscopic diagnosis and management of ampullary lesions. Gastrointest Endosc Clin N Am 23: 95-109. [Crossref]

6. Chua TC, Saxena A (2010) Extended pancreaticoduodenectomy with vascular resection for pancreatic cancer: a systematic review. J gastrointest Surg 14: 1442-1452. [Crossref]

7. Makary MA, Winter JM, Cameron JL, Campbell KA, Chang D, et al. (2006) Pancreaticoduodenectomy in the very elderly. $J$ Gastrointest Surg 10: 347-356. [Crossref]

8. Harewood GC, Pochron NL, Gostout CJ (2005) Prospective, randomized, controlled trial of prophylactic pancreatic stent placement for endoscopic snare excision of the duodenal ampulla. Gastrointest Endosc 62: 367-370. [Crossref]

9. Napole' on B, Gincul R, Ponchon T, Berthiller J, Escourrou J, et al. (2014) Endoscopic papillectomy for early ampullary tumors: long-term results from a large multicentre prospective study. Endoscopy 46:127-134. [Crossref]

10. Irani S, Arai A, Ayub K, Biehl T, Brandabur JJ, et al. (2009) Papillectomy for ampullary neoplasms: results of a single referral center over a 10-year period. Gastrointest Endosc 70: 923-932. [Crossref]

11. Kang SH, Kim KH, Kim TN, Jung MK, Cho CM, et al. (2017) Therapeutic outcomes of endoscopic papillectomy for ampullary neoplasms: retrospective analysis of a multicenter study. BMC Gastroenterology 17: 69. [Crossref]

12. Ridtitid, Tan D, Schmidt SE, Fogel EL, McHenry L, et al. (2014) Endoscopic papillectomy: risk factors for incomplete resection and recurrence during long-term follow-up. Gastrointest Endosc 79(2): 289-296. [Crossref]

Copyright: (C2018 Ruhua Z. This is an open-access article distributed under the terms of the Creative Commons Attribution License, which permits unrestricted use, distribution, and reproduction in any medium, provided the original author and source are credited. 
Ruhua Z (2018) A novel hemoclip applied on patients with duodenal papillary neoplasms during endoscopic papillectomy to prevent post-procedure bleeding 